Commun. math. Phys. 24, 303-309 (1972)

(C) by Springer-Verlag 1972

\title{
Superpropagators of Non-Localizable Fields
}

\author{
R. FLUME \\ II. Institut für Theoretische Physik der Universität Hamburg, Germany
}

Received November 3, 1971

\begin{abstract}
Arguments are given for a special choice of the superpropagator corresponding to Wightman-two-point-functions with an increase in momentum space as $e^{\kappa\left|p^{2}\right|^{1 / 2}}, \kappa>0$.
\end{abstract}

\section{Introduction}

Field theories with non-polynomial Lagrangians can be divided into two classes. The first class, which has been investigated by Jaffe [1], is characterized by the requirement that the increase of the vacuum expectation values (V.E.V.) in momentum space $\left.{ }_{0} L(1) \ldots L(n)_{0}\right\rangle^{1}$ is bounded by an indicator $g(t)$ with

$$
\int_{0}^{\infty} \frac{\log \left(g\left(t^{2}\right)\right)}{1+t^{2}} d t<\infty \quad(\text { see }[1])
$$

This is the necessary and sufficient condition in order that the admissible configuration space test-functions for the V.E.V. are dense in $D$, the space of $C^{\infty}$ functions having compact support. The second class contains all cases in which the growth condition is not fullfilled, i.e., especially those Lagrangians whose Wightman-two-point-functions grow as $\exp \left(\kappa\left|p^{2}\right|^{1 / 2}\right)$ or faster.

Given the Wightman functions $\left.{ }_{0} L(1) \ldots L(n)_{0}\right\rangle$ the problem of renormalization consists in the definition of time ordered V.E.V.'s $\left\langle{ }_{0} T(L(1) \ldots L(n))_{0}\right\rangle$. For a scalar field $\phi$ with exponential self-interaction $L=g: \exp (f \phi)-1:$, which belongs to Jaffe's class, Lehmann and Pohlmeyer [2] justify a special choice of the time-ordered two-pointfunction, the so called superpropagator $i\left\langle_{0} T(L(1) L(2))_{0}\right\rangle$. Their choice is uniquely distinguished from all other possibilities by a decrease of the real part of the superpropagator for $p^{2} \rightarrow \infty$ (if $f^{2}>0$ ). This property

${ }^{1} L(i)=L\left(\phi\left(x_{i}\right)\right)$, the interaction Lagrangian, may, in general, be a nonpolynomial function of several normal ordered fields. In this paper a function of a single scalar massless field is treated. The considerations of the second section apply, however, to the general case. 
is brought into connection with a certain kind of regularity of the real part at the zero point in configuration space and the choice is, therefore, called the "minimal singular" one.

In this paper arguments, deduced partially from [2], are given for a special choice of the superpropagator of a Lagrangian $L(\phi)$ whose Wightman-two-point-function $\left.{ }_{0} L(1) L(2)_{0}\right\rangle$ is of order $1 / 2$ and finite type as entire function in $p^{2}$ (that is, $\left\langle{ }_{0} L(1) L(2)_{0}\right\rangle=\delta^{4}\left(p_{1}+p_{2}\right) \Theta\left(\left(p_{1}\right)^{2}\right)$ $\Theta\left(p_{1}^{0}\right) F\left(p_{1}^{2}\right)$, const $\left.\left|F\left(p_{1}^{2}\right)\right|<e^{\kappa\left|p^{2}\right|^{1 / 2}}, \kappa>0, p^{2} \equiv p_{1}^{2}\right)$.

This case lies outside Jaffe's class, but with regard to increase properties in momentum space it is in the immediate neighbourhood of this class.

\section{Uniqueness Conditions for the Definition of a Superpropagator}

The imaginary part of a superpropagator is fixed by unitarity. The ambiguity enters the definition through the indeterminacy of the real part:

$$
\begin{aligned}
\operatorname{Re} i\left\langle_{0} T\{L(1) L(2)\}_{0}\right\rangle^{\star} & =\frac{1}{2}\left\langle_{0} \varepsilon\left(x_{1}^{0}-x_{2}^{0}\right)[L(1), L(2)]_{0}\right\rangle \\
& =\mathscr{F}^{-1}\left(\delta^{4}\left(p_{1}+p_{2}\right)\left\{F\left(p_{1}^{2}\right)+G\left(p_{1}^{2}\right)\right\}\right) .
\end{aligned}
$$

The ambiguity is represented by the arbitrary entire function $G\left(p^{2}\right)$ of order $\leqq \frac{1}{2}$ in $p^{2}$.

On the definition of $\varepsilon$-commutator V.E.V.'s (e.C.V.) two conditions are imposed:

$\alpha$ ) The $\varepsilon . C . V$. shall decrease for $p^{2} \rightarrow \infty$ (alternatively for $p^{2} \rightarrow-\infty$ ).

$\beta$ ) The increase of the $\varepsilon . C . V$. for $p^{2} \rightarrow-\infty$ (resp. $p^{2} \rightarrow \infty$ ) shall be bounded by a Jaffe-indicator $g(t)$ mentioned above.

Suppose one has found a definition of the $\varepsilon$.C.V. which fullfills $\alpha$ and $\beta$, then every addition of an entire function $G\left(p^{2}\right)$ of order $1 / 2$ in $p^{2}$ with a decrease in one direction - according to prescription $\alpha$ - will increase in the opposite direction stronger than every Jaffe-indicator (which can easily be shown with the help of the Phragmén-Lindelof-theorem). The prescriptions $\alpha$ and $\beta$, therefore, make the definition of the $\varepsilon$.C.V. unique.

Prescription $\alpha$ can be justified with the principle of least singularity as in [2]. Prescription $\beta$ is the weakest condition to be imposed in order to get a "sharp" statement on local properties of the $\varepsilon . C . V$. in a sense made clear below.

The $\varepsilon . C . V$. applied to a test-function $\psi$, which is analytic in a neighbourhood of the lightcone, can be written (independent of the special

$\star$ In the following the factor $\delta\left(p_{1}+p_{2}\right)$ will be dropped; $p_{1}^{2} \equiv p^{2}$. (F्F stands for the Fourier transform). 
definition of the $\varepsilon . C . V$.$) as follows:$

$$
\begin{aligned}
\left(\left\langle_{0} \varepsilon[L(1), L(2)]_{0}\right\rangle, \psi(1-2)\right) \\
=\left(\delta^{(1)}\left((z)^{2}\right), \tilde{\psi}_{1}(z)\right)+\tilde{\psi}_{2}(0), 1-2 \equiv z \\
\quad \tilde{\psi}_{i}(z)=\oint_{|\xi|=l} d \xi W_{i}(\xi) \int_{\|\varrho\| \leqq 1} d^{4} \varrho \psi\left(z_{0}+i \varrho_{0} \xi, z+\varrho \xi\right), i=1,2 \\
\quad \delta^{(1)}\left((z)^{2}\right) \sim \mathscr{F}\left(\ln \left|p^{2}\right|\right) .
\end{aligned}
$$

The respresentation (3) can be derived from the following identity (see [3]):

$$
\begin{aligned}
& \left(\Sigma \frac{c_{n}}{(2 n) !} \square^{n} \delta^{4}\left(x-x^{\prime}\right), \psi(x)\right) \\
& =\oint_{|\xi|=l} W(\xi) d \xi \int_{\|\varrho\| ! 1} d^{4} \varrho \psi\left(x_{0}^{\prime}+i \varrho_{0} \xi, x^{\prime}+\varrho \xi\right) \\
& W(\xi)=\frac{1}{2 \pi i} \sum_{n=0}^{\infty} \frac{c_{n} 2^{2 n} n !(n+2) !}{\pi(2 n) ! \xi^{2 n+1}}
\end{aligned}
$$

$W(\xi)$ is holomorphic outside a finite region $|\xi|>l-\delta$ and Eq. (4) is correct whenever $\sum_{0}^{\infty} \frac{c_{n}\left(p^{2}\right)^{n}}{(2 n) !}$ is an entire function of order $\leqq \frac{1}{2}$ in $p^{2}$. The coefficients $c_{n}$ involved in (3) fullfill this requirement.

Suppose now that a special definition of the $\varepsilon$.C.V. has the properties $\alpha$ and $\beta$. Then $\phi \in D^{4}$ exists which belongs to the translation invariant domain of test-functions of the $\varepsilon$.C.V. Let $\phi=0$ for $x^{2}>-l-\delta, \delta>0$.

Then $\left(\left\langle_{0} \varepsilon[L(1), L(2)]_{0}\right\rangle, \phi(1-2)\right)=0$ because $\phi$ can be approximated by functions $\psi_{n}$ which, on the one hand, are analytic in the region

$$
g=\left\{y ; y=z^{\mu}+\left(i \varrho_{0} \xi, \varrho \xi\right),\|\varrho\| \leqq 1,|\xi| \leqq l,(z)^{2}=0\right\}
$$

and converge there to zero sufficiently rapidly; on the other hand, they approximate $\phi$ in the dual topology of the Jaffe-class test-functions.

$$
\left(\left\langle_{0} \varepsilon[]_{0}\right\rangle, \phi\right)=\lim _{V \rightarrow \infty}\left(\left\langle_{0} \varepsilon[]_{0}\right\rangle, \psi_{V}\right)=0 .
$$

The last equality follows from the representation (3).

Closely connected with its local properties is a kind of regularity of the $\varepsilon . C . V$. at $x=0$. If the $\varepsilon . C . V$. decreases, at least as $1 / p^{2}$ for $p^{2} \rightarrow \infty$, then it can also be written as a limit of a shrinking procedure:

$$
\left\langle_{0} \varepsilon[]_{0}\right\rangle=\lim _{t \rightarrow 0} \chi_{t}(x)\left\langle_{0} \varepsilon[]_{0}\right\rangle
$$

where $\chi_{t}(x)$ is a suitable multiplicator function:

$$
\chi_{t}(x)= \begin{cases}1, & \left|x_{0}\right| \geqq t \\ 0, & \left|x_{0}\right| \leqq \frac{t}{2}\end{cases}
$$


For an addition to the $\varepsilon$.C.V. consisting of an entire function $G\left(p^{2}\right)$ of order $1 / 2$ in $p^{2}$ which also decreases as $1 / p^{2}$ for $p^{2} \rightarrow \infty$, the shrinking would be possible only in a more restrictive sense. Whereas for the $\varepsilon . C . V$. the multiplicators can be chosen in such a way that they vanish beyond a finite distance from the zero point (the E.C.V., obeying prescriptions $\alpha$ and $\beta$, belongs to the Jaffe-class distributions), the multiplicators of $\mathscr{F}\left(G\left(p^{2}\right)\right)$ must not be chosen to decrease in the direction of the space coordinates too rapidly, so that $\mathscr{F}(G) \chi_{t}$ makes sense and the limit $t \rightarrow 0$ exists.

\section{The E.C.V. of the Lagrangian}

$$
L^{\prime}(\phi)=g \int_{0}^{\infty} \frac{d y}{y} e^{-y^{2}}: e^{y f \phi}-1:
$$

The above quoted interaction Lagrangian $L^{\prime}(\phi)$ is taken $(\phi$ is a scalar massless field) as an example where the previously given prescriptions lead to a definite choice of the $\varepsilon . C . V$. Two equivalent representations of the $\varepsilon . C . V$. are considered.

The first, due to Volkov [4], is given by a Sommerfeld-Watson integral:

$$
\begin{aligned}
& \frac{1}{g^{2}}\left\langle{ }_{0} \varepsilon[,]_{0}\right\rangle \\
& =4 \pi^{2} \quad \int d z \frac{\left(p^{2}\right)^{z-2} \Gamma^{2}(z / 2) \lambda^{z}(1 / 4)^{z}}{\sin ^{2} \pi z \Gamma(z-1) \Gamma(z) \Gamma(z+1)} \quad \lambda=\frac{f^{2}}{4 \pi^{2}} \\
& \quad \oint \underset{+}{\longrightarrow}
\end{aligned}
$$

for $p^{2}>0$.

The decrease for $p^{2} \rightarrow \infty$ is obvious, because the path of integration can be deformed to lie parallel to the imaginary axis. For $\left.p^{2}<0<_{0} \varepsilon[]_{0}\right\rangle$ can be represented as follows:

$$
\begin{aligned}
\frac{1}{g^{2}} & \left\langle{ }_{0} \varepsilon[]_{0}\right\rangle \\
& =4 \pi^{2} \int d z \frac{\cos \pi z\left|p^{2}\right|^{z-2} \Gamma^{2}(z / 2) \lambda^{z}(1 / 4)^{z}}{\sin ^{2} \pi z \Gamma(z-1) \Gamma(z) \Gamma(z+1)} \\
& =4 \pi^{2} \int_{0}^{1} d t \underset{+}{\longrightarrow} d z \frac{\cos \pi z t^{\frac{z-2}{2}}(1-t)^{\frac{z-2}{2}}\left|p^{2}\right|^{z-2} \lambda^{z}(1 / 4)^{z}}{\sin ^{2} \pi z \Gamma(z-1) \Gamma(z+1)} .
\end{aligned}
$$

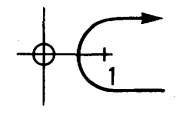


The Meijer- $G$-function corresponding to the $z$-integration is bounded for $0<t<1$ and $\left|p^{2}\right| \rightarrow \infty$ (see Luke [5]) and, therewith, also the $\varepsilon . C . V$. is bounded.

The second representation is obtained if one superposes "minimal singular" exponential $\varepsilon . C . V$. .'s according to [2].

$$
\begin{gathered}
\left\langle{ }_{0} \varepsilon[,]_{0}\right\rangle=g^{2} \int_{0}^{\infty} \int_{0}^{\infty} \frac{d y}{y} \frac{d y^{\prime}}{y^{\prime}} e^{-\left(y^{2}+y^{\prime} 2\right)} \\
\left\langle{ }_{0} \varepsilon\left[: e^{f \phi y}-1:,: e^{f \phi^{\prime} y^{\prime}}-1:\right]_{0}\right\rangle, \quad \text { Lehmann, Pohlmeyer. }
\end{gathered}
$$

To see that (5) and (6) are identical, one can either directly insert in (6) the expansion of $\left\langle_{0} \varepsilon\left[: e^{f \phi y}-1: e^{f \phi^{\prime} y^{\prime}}-1:\right]_{0}\right\rangle_{\mathscr{L}, \mathscr{P}}$. in powers of $y y^{\prime} f^{2}$ and show that the $y$-integrations are interchangeable with the summation which leads to (5), or one can note that the $\varepsilon . C . V$. for Lagrangians

$$
L^{\prime}(\phi, \lambda)=G \int e^{-y^{2 \lambda}} 1 / y: e^{y f \phi}-1: d y
$$

defined analogously to (5) and (6) are analytic in $\lambda$ in a region which contains $\lambda=1$ (at least on test-functions with compact support in momentum space). For $\operatorname{Re} \lambda>1 L^{\prime}(\lambda)$ belongs to the Jaffe-fields and both representations of the $\varepsilon$.C.V. there satisfy the uniqueness condition of decreasing for $p^{2} \rightarrow \infty$.

It has been shown in [2] that the "minimal singular" $\varepsilon . C . V$. of the exponential Lagrangian can also be obtained from a shriking procedure:

$$
\left\langle_{0} \varepsilon\left[: e^{f \phi}-1:,: e^{f \phi^{\prime}}-1:\right]_{0}\right\rangle=\lim _{t \rightarrow 0}\left\langle_{0} \varepsilon\left[: e^{f \phi}-1:,: e^{f \phi^{\prime}}-1\right]_{0}\right\rangle \chi_{t}(x)
$$

$\chi_{t}(x)$ being a multiplicator with

$$
\chi_{t}(x)= \begin{cases}1, & \left|x_{0}\right| \geqq t \\ 0, & \left|x_{0}\right| \leqq \frac{t}{2}\end{cases}
$$

whose space derivatives on the lightcone are bounded. That this shrinking is possible is due to the fact the commutator V.E.V. (C.V.) smeared out in the space variables is a $C^{\infty}$ function of the time variable outside $x_{0}=0$, and has finite limits if one approaches from either side to $x_{0}=0$. This property is conserved for the C.V. of $L^{\prime}(\lambda)$ if $\operatorname{Re} \lambda \geqq 1$ but no longer if $\operatorname{Re} \lambda<1$. This results from the following estimates ${ }^{2}$ :

\footnotetext{
${ }^{2}$ Cf. Lehmann, Pohlmeyer [6].
} 
Let $\tilde{\psi}(s)=\int d \Omega_{3} \psi(\boldsymbol{x})_{\|\boldsymbol{x}\|^{2}=s}$ be analytic in $s$.

$$
\begin{aligned}
& \left|\int d^{3} x<_{0}\left[: e^{y f \phi(1)}-1:,: e^{y^{\prime} f \phi(2)}-1:\right]_{0}\right\rangle \psi(\boldsymbol{x}) \mid \\
& \left(1^{0}-2^{0}=x^{0}, \mathbf{1}-\mathbf{2}=\boldsymbol{x}\right)
\end{aligned}
$$

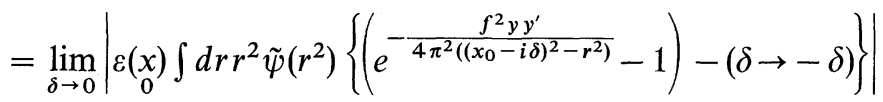

$$
\begin{aligned}
& =\left|\frac{1}{2} \quad \int \quad d \phi \sqrt{x_{0}^{2}-\phi} \tilde{\psi}\left(x_{0}^{2}-\phi\right)\left(e^{-\frac{f^{2} y y^{\prime}}{4 \pi^{2} \phi}}-1\right)\right| \\
& \$=\mathbb{C}^{1} \\
& \boldsymbol{x}_{0} \rightarrow 0\left|\frac{1}{2} \int_{\mathfrak{C}^{1}} d \phi \sqrt{-\phi} \tilde{\psi}(-\phi)\left(e^{-\frac{f^{2} y y^{\prime}}{4 \pi^{2} \phi}}-1\right)\right| \\
& <2 \int_{0}^{f^{2} / 4 \pi^{2}}|\sqrt{-\phi} \tilde{\psi}(-\phi)| d \phi \\
& +\oint_{|\phi|=f^{2} / 4 \pi^{2}} d|\phi||\tilde{\psi}(-\phi) \sqrt{-\phi}|\left(1+e^{y y^{\prime}}\right) .
\end{aligned}
$$

The last estimate implies

$$
\begin{aligned}
\lim _{x_{0} \rightarrow 0} \mid & \mid \int_{0}^{\infty} \int_{0}^{\infty} \frac{d y}{y} \frac{d y^{\prime}}{y^{\prime}} e^{-\left(y^{2 \lambda}+y^{\prime 2 \lambda}\right)} \\
& \left.\cdot \int d^{3} x<_{0} \varepsilon\left[: e^{y f \phi(1)}-1:,: e^{y^{\prime} f \phi(2)}-1:\right]_{0}\right\rangle, \psi(\boldsymbol{x}) \mid<\infty
\end{aligned}
$$

because

$$
\left|\int_{0}^{\infty} \int_{0}^{\infty} d y d y^{\prime} e^{-\left(y^{2 \lambda}+y^{\prime 2 \lambda}\right)+y y^{\prime}}\right|<\infty
$$

for $\operatorname{Re} \lambda \geqq 1$.

The preceding considerations show that the special definition (6) of the E.C.V. is connected with the (unique) extension C.V. of the C.V. to those test-functions which are analytic in the space coordinates and vanish in a neighbourhood of $x_{0}=0$.

Let $\psi_{t}(x)=\chi_{t}\left(x_{0}\right) \psi(x), \psi(x) \in Z=\mathscr{F}\left(D^{4}\right), \chi_{t}\left(x_{0}\right)$ as in (7), but only dependent on $x_{0}$

$$
(\varepsilon . \mathrm{C} . \mathrm{V} ., \psi)=\lim _{t \rightarrow 0}\left(\overline{\mathrm{C} . \mathrm{V}} ., \varepsilon\left(x_{0}\right) \psi_{t}\right) .
$$

By application of a shrinking $\lim _{t \rightarrow 0} \chi_{t}(x)$ C.V. a functional is discarded from the C.V. which for $\operatorname{Re} \lambda>1$ is concentrated on $x=0$ and for $\lambda=1$ on a finite complex neighbourhood of $x=0$. 
One has to note that relation (8) between commutator and $\varepsilon$-commutator is of a formal nature, because a Lorentz-transformation of the extension of the C.V. is not defined.

Acknowledgement. I am indebted to Professor H. Lehmann for many discussions and I thank Dr. K. Pohlmeyer for several hints.

\title{
References
}

1. Jaffe, A.: High energy behaviour of strictly localizable fields II. (Unpublished manuscript).

2. Lehmann, H., Pohlmeyer, K.: Commun. math. Phys. 20, 101 (1971).

3. Efimov, G. V.: Commun. math. Phys. 7, 138 (1968).

4. Luke,Y.L.: The special functions and their approximations, Vol. I, p. 192, Theorem 5. New York: Academic Press 1969.

5. Volkov, M. K.: Ann. Phys. 49, 202 (1968).

6. Lehmann, H., Pohlmeyer, K.: Perturbation theory for a class of nonpolynomial lagrangian field theories. Coral Gables Conference 1971.

\author{
R. Flume \\ II. Institut für Theoretische Physik \\ der Universität \\ D-2000 Hamburg 50 \\ Luruper Chaussee 149 \\ Germany
}

